\title{
Electric Field Control of Magnetic Coupling in a Double Quantum Dot System and Related Parasitic Electric Dipole Effect
}

\author{
Z. BAK* \\ Institute of Physics, Jan Długosz University of Częstochowa, al. Armii Krajowej 13/15, 42-200 Częstochowa, Poland
}

\begin{abstract}
We prove that tunable magnetic interaction in a two-qubit spintronic device can arise due to the mutual competition of the Ruderman-Kittel-Kasuya-Yosida and double-exchange interactions. The values of induced electric dipoles (which arise when magnetic coupling is manipulated by the electric field) are calculated. We show that the dissipation of the energy during logic operations due to these parasitic dipoles can destroy quantum coherence in any quantum dot system.
\end{abstract}

PACS numbers: 73.21.La, 73.90.+f, 75.75.Lf

\section{Magnetic interactions}

One of the possible realizations of quantum computing is based on a system in which a qubit represents quantum information by spin orientation of the solid state cell. The simplest case of such a cell contains two magnetically coupled quantum dots with electron spins trapped in them. Logical operations on such a qubit require modulations of the interdot magnetic exchange [1]. We show that under some conditions, such modulation can be achieved by mutual competition of the RudermanKittel-Kasuya-Yosida (RKKY) interaction and voltage operated double-exchange (DE) interaction. We point out that switching off magnetic interaction by an electric field induces parasitic instantaneous electric dipoles. We prove that the dissipation of the energy during logic operations due to these parasitic dipoles can destroy quantum coherence in the spintronic systems.

Electrons (both from a lead and/or from the metallic substrate) scattered off QD spins induce effective spin exchange interaction, the RKKY interaction [2]. The exchange integrals of the RKKY coupling depend on the density of the free electron states $n(\epsilon)$ (in vicinity of the Fermi energy). In many confined electron systems the density of states is given by $n(\epsilon) \mathrm{d} \epsilon \approx\left(\epsilon-\epsilon_{0}\right)^{\alpha / 2-1} \mathrm{~d} \epsilon$. The parameter $\alpha$ represents the effective spectral dimension which in many cases, is a fraction [3]. The RKKY exchange integral in the case is given by [4]:

$$
\begin{aligned}
& J(\xi)=J_{0} \xi^{\alpha-2}\left[J_{\alpha / 2-1}(\kappa \xi) Y_{\alpha / 2-1}(\kappa \xi)\right. \\
& \left.\quad+J_{\alpha / 2}(\kappa \xi) Y_{\alpha / 2}(\kappa \xi)\right],
\end{aligned}
$$

The fractional dimension [4] can arise also in our system.

Let us consider a double quantum dot system (DQD)

* e-mail: z.bakakd.czest.pl. in the lateral configuration joined by a lead. There can arise two utmost regimes of mutual electron exchange, namely the weak coupling regime (in case when the dot-lead coupling dominates the direct dot-dot hopping) and its alternative. In the weak coupling regime electrons are localized on an individual QD and in this case both quantum dots $\mathrm{QD}_{1}$ and $\mathrm{QD}_{2}$ can be considered separately. When the opposite limit is realized the strong interdot binding causes that a DQD can be treated as an artificial molecule/dimer. Evidently the real DQDs are in between these two utmost limits.

It is a well known fact that by a change of the gate voltage applied to a QD a mixed-valent behavior can be generated. Suppose that a mixed valent $\mathrm{QD}_{1}-\mathrm{QD}_{2}$ dimer can exist in either the $\mathrm{QD}_{1}^{* *}-\mathrm{QD}_{2}^{*}$ or $\mathrm{QD}_{1}^{*}-\mathrm{QD}_{2}^{* *}$ electronic configurations, where lower index numbers the QDs, while stars denote the number of electrons localized on the dot. Provided that the $\mathrm{QD}_{1}-\mathrm{QD}_{2}$ dimer enters the mixed-valent behavior the correlated hopping of electrons between QDs gives rise to ferromagnetic (double-exchange) interaction between QD spins [5]. It is important to note that a single electron that hops between two magnetic QDs suffices to form "double exchange" coupling. Contrary to the RKKY coupling which (with interdot separation) oscillates between ferro- and antiferromagnetic, the DE coupling is always ferromagnetic. As the strength of double-exchange is governed by the interdot hopping, which in turn depends on the gate voltages, one can manipulate it from outside. This effect offers us a new possibility of control and tunability for molecular spintronics.

Suppose the interdot separation fulfills the condition for antiferromagnetic RKKY coupling. By switching the voltage on or off there arises voltage operated (ferromagnetic) double-exchange interaction. The effective magnetic coupling between QD spins is thus the superposition of antiferromagnetic RKKY and voltage operated (ferro- 
magnetic) double-exchange. This possibility allows us to create either ferro- or antiferro-correlation between QDs spins, being the prerequisite for construction of universal quantum logic gates [1].

\section{Electric field effects}

Usually when dealing with the QDs the spherical symmetry is assumed, which is due to the fact that electronic structure of such a QD mimics that of the hydrogen atom. However, the most common method of QD fabrication is based on deposition of metal/semiconducting components on a flat substrate. This results in formation of a disc-like QD with lateral extension much exceeding its thickness. Therefore the electron mobility can be assumed as two-dimensional, while the electron in dot confinement with a good approximation can be modeled by a parabolic potential. With the assumptions above the electron Hamiltonian can be written in a form which resembles a two-dimensional harmonic oscillator [6]. During quantum logic operations there arises contribution from the external electric field. Suppose we apply the electric field $F$ along " $x$ "-axis, then we have

$$
H=\left(p_{x}^{2}+p_{y}^{2}\right) /\left(2 m_{\mathrm{e}}\right)+m_{\mathrm{e}} \omega_{0}^{2}\left(x^{2}+y^{2}\right) / 2+e F x,
$$

where $m_{\mathrm{e}}$ is the effective mass of an electron while $\omega_{0}$ is the strength of the parabolic confinement. The electron eigenstates in our dot are given by the products of the eigenfunctions of the one-dimensional shifted harmonic oscillator [6]:

$$
\begin{aligned}
& \Psi_{n_{x} n_{y}}(x, y)=\Phi_{n_{x}}(x+\beta) \Phi_{n_{y}}(y), \\
& n_{x}, n_{y}=0,1, \ldots
\end{aligned}
$$

where $\Phi_{n_{i}}\left(x_{i}\right)=A_{n} \exp \left(-\alpha^{2} x_{i}^{2}\right) H_{n_{i}}\left(\alpha x_{i}\right), x_{i}=x, y$, with $H_{n_{i}}\left(\alpha x_{i}\right)$ being the Hermite polynomials while $\alpha=$ $\sqrt{m_{\mathrm{e}} \omega_{0} / \hbar}$. The shift of the electron cloud center $\beta$ equals $\beta=e F /\left(m_{\mathrm{e}} \omega^{2}\right)$. Evidently any electric field $\boldsymbol{F}$ applied to a QD (e.g., during logic operations) not only shifts the electron energy levels but also induces spontaneous electric dipole moments. The electric dipole $\boldsymbol{p}$ induced in a disc-like QD of radius $R_{\mathrm{D}}$ with an electron being in the state $\Psi_{n_{x} n_{y}}(x, y)$ by the electric field equals $p=e \beta$. In the following we assume that the electric field $\boldsymbol{F}$ arises due to the gate voltage $V$, i.e, $F=V / R_{\mathrm{D}}$. The spontaneous electric dipoles $p_{1}$ and $p_{2}$ of both QDs interact with each other and the instantaneous dipole moments vary when the gate voltage is changed. This generates dissipation of energy and may lead to overheating of the spintronic device.

Let us estimate the energy associated with the two instantaneous dipole-dipole interactions. The dipoledipole interaction energy is of order of $E \approx p^{2} /\left(\epsilon_{0} D^{3}\right)$, where $D$ is the distance between centers of two adjacent QDs and $p$ - the induced dipole moment. Estimation of the induced dipole moment goes as follows. Suppose we have two QDs of the diameter $D=20 \mathrm{~nm}$ with their centers separated by $D=20 \mathrm{~nm}$. The value of spontaneous dipole moment equals $p=e \beta=e^{2} F /\left(m_{\mathrm{e}} \omega^{2}\right)[6]$, i.e. electron charge times shift of the center of electron cloud within a QD. The electric field can be calculated from the values of the gate voltage $V_{\mathrm{g}}$ (we take $V_{\mathrm{g}}=0.5 \mathrm{~V}$ as in [7]). Thus from $F=V_{\mathrm{g}} / D$ one can immediately obtain $F=2.5 \times 10^{7} \mathrm{~V} / \mathrm{m}$. The value of the $m_{\mathrm{e}} \omega^{2}$ can be deduced from the value of the QD parabolic confinement potential $V_{\text {conf }}=m_{\mathrm{e}} \omega^{2} D^{2} / 8$. Taking the barrier height $V_{\text {conf }}$ of the parabolic DQ as 70 percent of the difference in a band gap between a QD and matrix materials [8] we obtain $V_{\text {conf }} \approx 0.2 \mathrm{eV}$ in the InAs/GaAs(matrix) system.

From this relation we obtain $p=e \beta=e^{2} F /\left(m_{\mathrm{e}} \omega^{2}\right) \approx$ $6 e \mathrm{~nm}$. Using the estimations above we can estimate the dipole-dipole interaction energy as $E=p^{2} /\left(\epsilon_{0} D^{3}\right) \approx$ $0.08 \mathrm{eV}$. It seems rather small but we must remember that qubits are assumed to be elements of the RAM memory and when the information will be written or erased the energy stored by parasitic instantaneous dipoles will be dissipated. The frequency of spin operations in the quantum computers must exceed frequency limited by the QD spin decoherence time. In the InAs/GaAs (matrix) based QD the spin decoherence time $T_{2}$ equals 2 ns [9], which means that the spin flipping frequency must be of order of $10^{9} \mathrm{~Hz}$. If we multiply the energy $E$ dissipated in a single flop process $E \approx 0.08 \mathrm{eV}$ by computing frequency $\omega_{\text {comp }}=10^{9} \mathrm{~Hz}$ we can see that dissipated energy in a second of computing time is sufficiently large to destroy quantum coherence of the spintronic cell.

The shift of the electron (spin) clouds is important since the RKKY interaction oscillates with the interspin separation favoring either ferro- or antiferromagnetic ordering. To estimate the change of interspin distance arising due to the applied fields, let us assume that $\boldsymbol{F}_{1}=-\boldsymbol{F}_{2}$, and the direction of applied fields is perpendicular to the lead which joins centers of both QDs. Under assumptions above we find that $d_{\mathrm{s}}^{\prime}=\left(d_{\mathrm{s}}^{2}+4 \beta^{2}\right)^{1 / 2}$. If we take into account that $\beta$ can reach the value of $6 \mathrm{~nm}$ we find that it is large enough to switch the RKKY integral from negative to positive (or vice versa). This effect offers the new possibility to control magnetic interactions.

\section{References}

[1] D. Stepanenko, N.E. Bonsteel, D.P. DiVincenzo, G. Burkard, D. Loss, Phys. Rev. B 68, 115306 (2003).

[2] P. Simon, R. Lopez, Y. Oreg, Phys. Rev. Lett. 94, 086602 (2005).

[3] Z. Bak, Phys. Rev. B 68, 064511 (2003).

[4] Z. Bak, R. Jaroszewicz, W. Gruhn, Acta Phys. Pol. A 115, 342 (2009).

[5] G.B. Martins, C.A. Busser, K.A. Al-Hassanieh, A. Moreo, E. Dagotto, Phys. Rev. Lett. 94, 026804 (2005).

[6] W. Xie, Q. Xie, Physica E 404, 1625 (2009).

[7] H. Drexler, D. Leonard, W. Hansen, J.P. Kotthaus, P.M. Petroff, Phys. Rev. Lett. 73, 2252 (1994).

[8] E.J. Peter, Phys. Lett. A 355, 59 (2006).

[9] S. Kashiwada, T. Matsuda, K. Yoh, J. Cryst. Growth 301-302, 785 (2007). 